\title{
CORRIGENDUM
}

\section{Modelling of peripheral fluid accumulation after a crystalloid bolus in female volunteers - a mathematical study}

\author{
Peter Rodhe, Dan Drobin, Robert G. Hahn, Bernt Wennberg, Christina Lindahl, \\ Fredrik Sjöstrand and Christer H. Svensen
}

The incorrect versions of Figures 1 and 2 and Tables 1 and 2 were published online for this article. Now included in this issue, the correct versions can be found below:
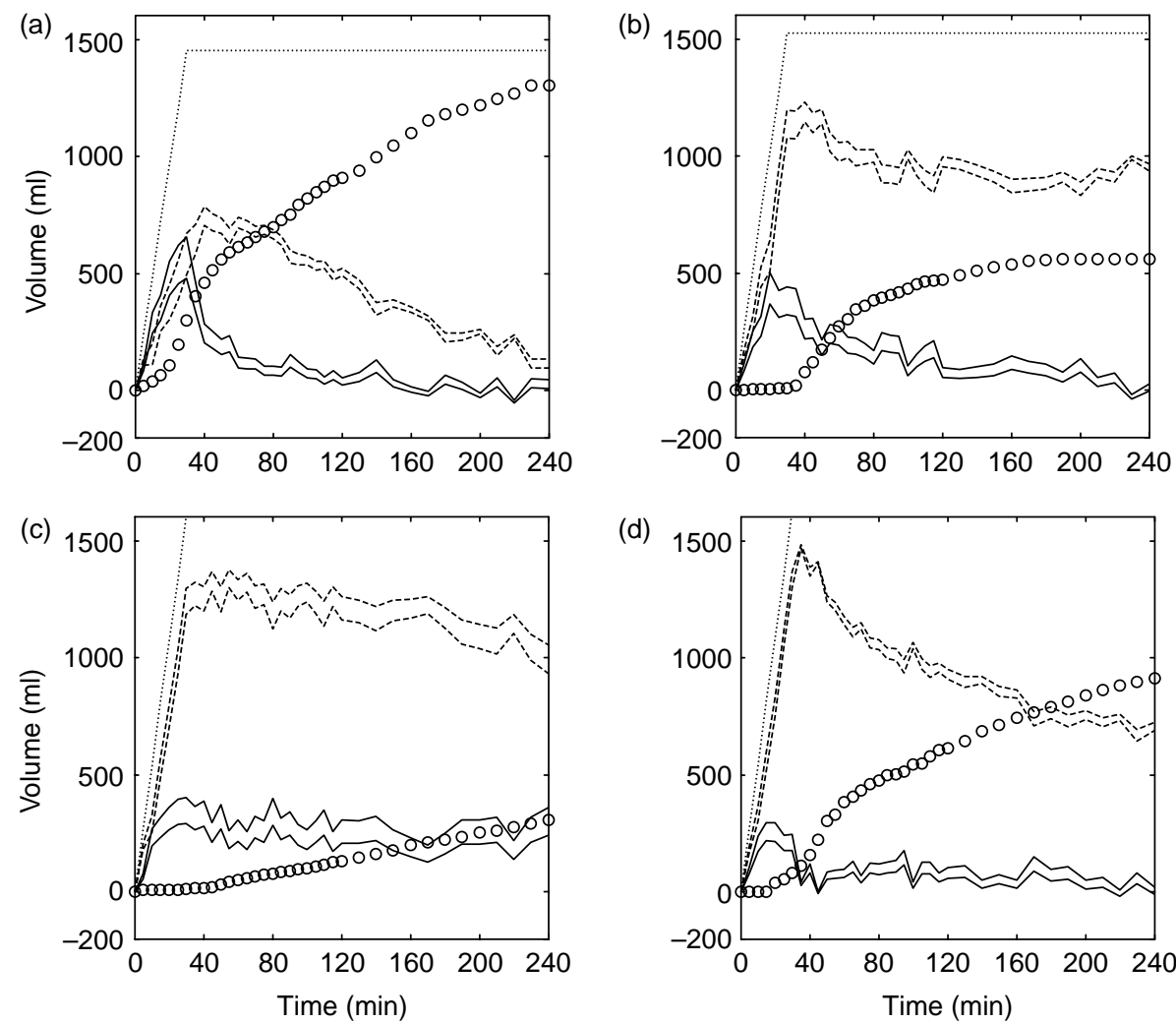

Figure 1. Empirical computation. Double bar lines show perturbated volumes of central (lower) and peripheral (upper) volumes respectively. Circle lines reflect urinary output. The small dotted line is infusion which ends at 30 minutes. 


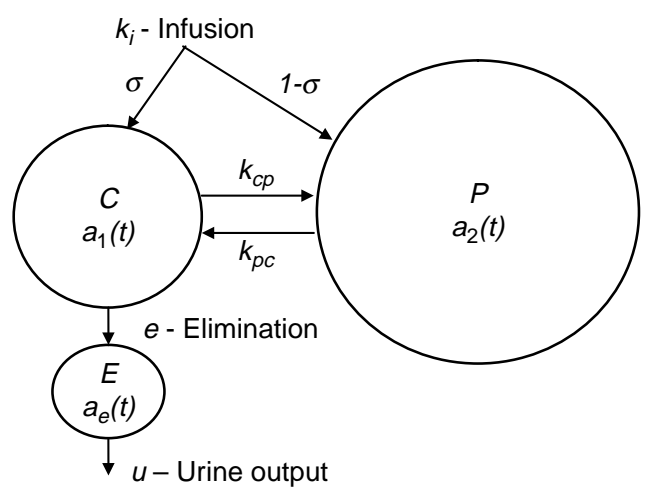

Figure 2. Overview of kinetic model. See text for definitions of symbols.

Table 1. Definition of model parameters.

\begin{tabular}{lll}
\hline & Unit & Description \\
\hline $\boldsymbol{k}_{\boldsymbol{t}}$ & $\mathrm{ml} / \mathrm{min}$ & Intercompartment rate constant \\
$\boldsymbol{k}_{r}$ & - & Dilution-dependent elimination parameter \\
$\boldsymbol{k}_{b}$ & $\mathrm{ml} / \mathrm{min}$ & Basal elimination constant \\
$\boldsymbol{\sigma}$ & - & Bypass factor \\
$\boldsymbol{\mu}$ & - & Distribution factor $v_{\mathrm{c}} / v_{p}$ \\
$\boldsymbol{T}_{l}$ & $\min$ & Delay time of elimination response \\
$\boldsymbol{T}_{e}$ & $\min$ & Turn-over time of intermediate compartment \\
$\boldsymbol{T}_{u}$ & min & Delay from intermediate compartment to $u_{\text {output }}$ \\
\hline
\end{tabular}

Table 2. Parameter estimates.

\begin{tabular}{lcc}
\hline$k_{t}$ & 114 & $(45-209)$ \\
$1 / \mu$ & 2.7 & $(2.0-5.7)$ \\
$\sigma$ & 0.69 & $(0.56-0.87)$ \\
$V_{\sigma}$ & 372 & $(184-694)$ \\
$k_{e l}$ & 21.4 & $(9.3-34.3)$ \\
$k_{b}$ & 1.11 & $(0.28-2.90)$ \\
$E_{e f f}$ & 24 & $(5-35)$ \\
$T_{e}$ & 10.6 & $(4.9-20.9)$ \\
$T_{l}$ & 1.4 & $(0.8-3.4)$ \\
$T_{u}$ & 4.2 & $(2.3-7.2)$ \\
\hline
\end{tabular}




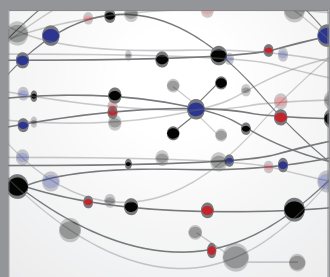

The Scientific World Journal
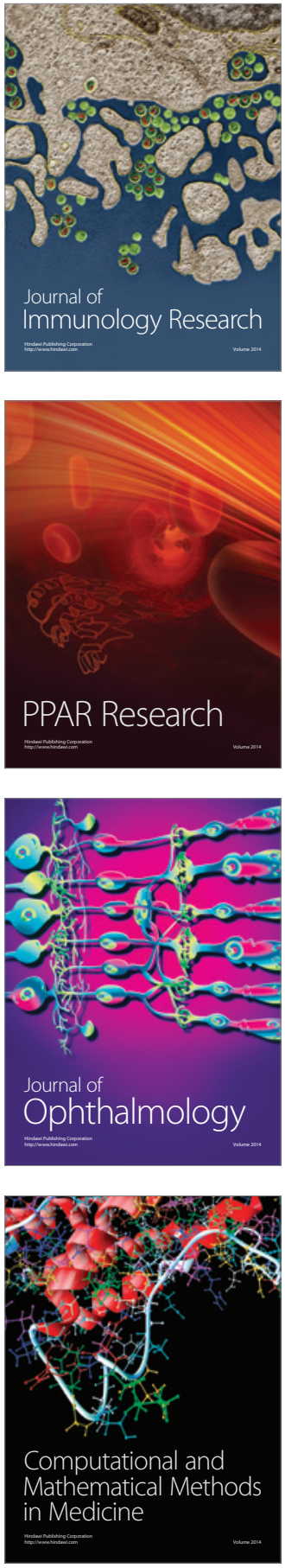

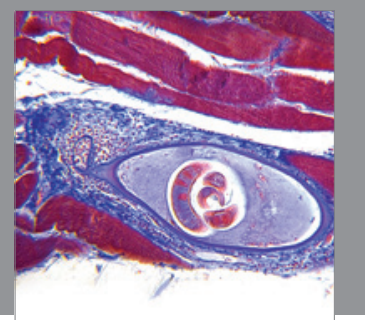

Gastroenterology

Research and Practice
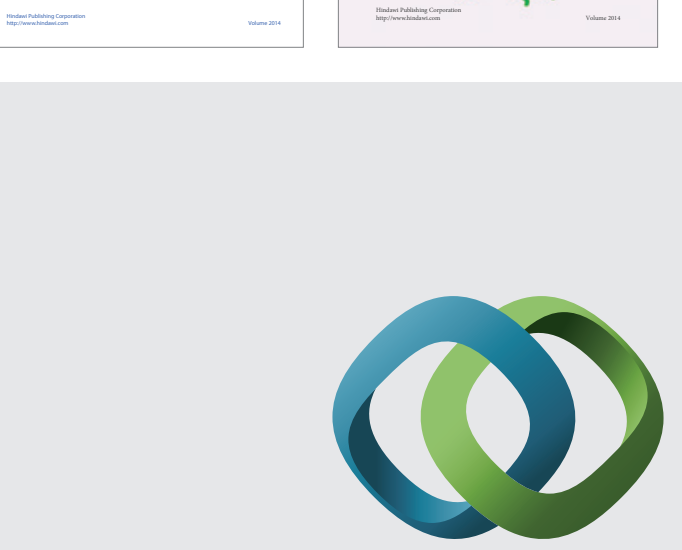

\section{Hindawi}

Submit your manuscripts at

http://www.hindawi.com
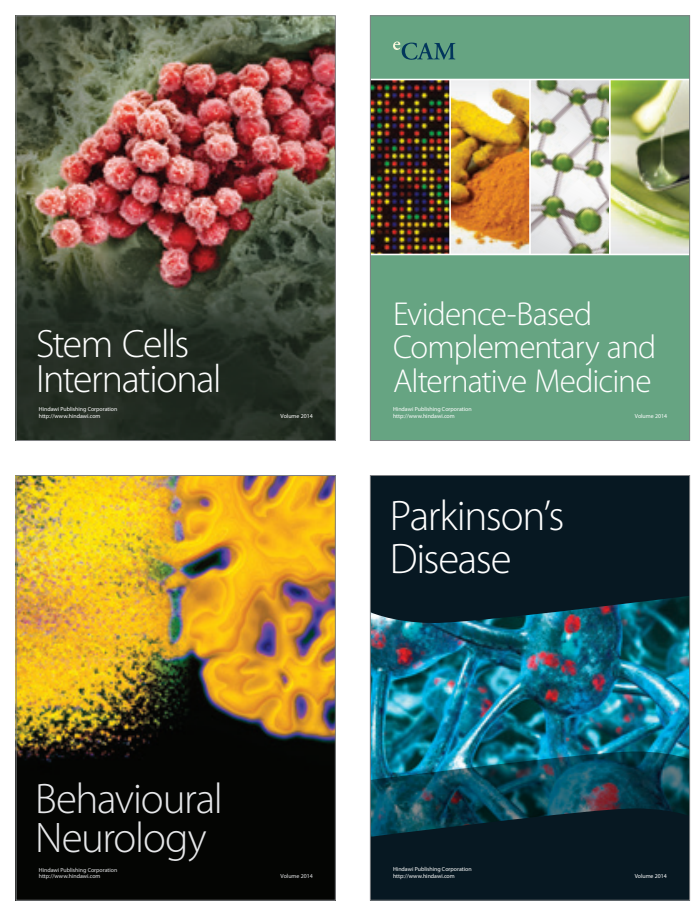

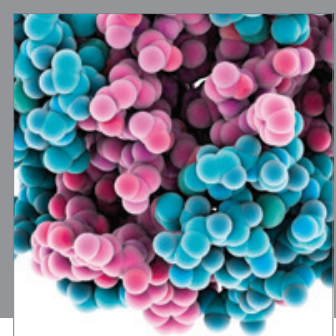

Journal of
Diabetes Research

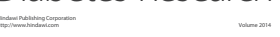

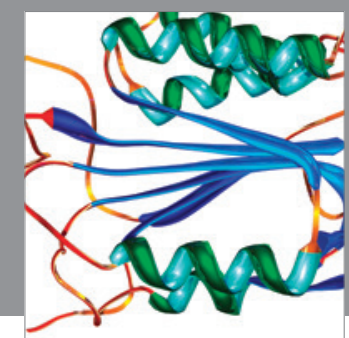

Disease Markers
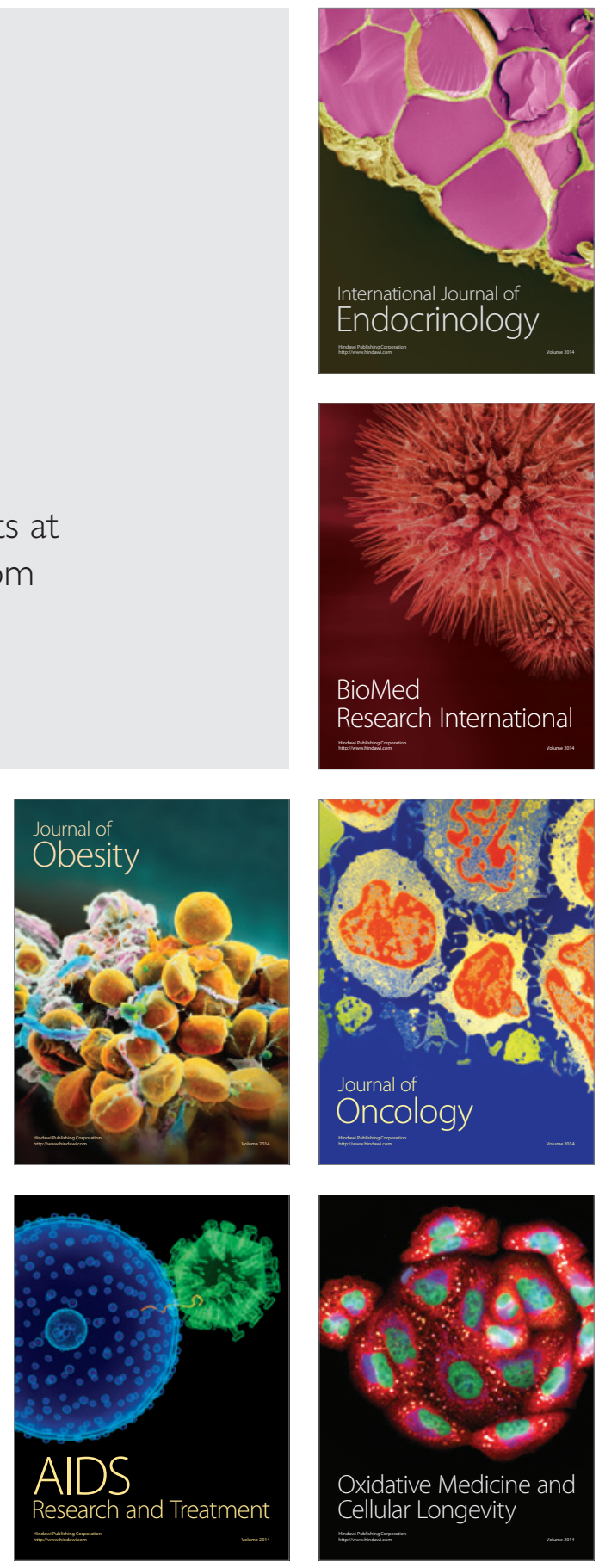